\title{
Special issue on bacterial citrus diseases: part II
}

\author{
Alessandra Alves de Souza ${ }^{1} \cdot$ Nian Wang $^{2} \cdot$ Helvecio Della Coletta-Filho ${ }^{1} \cdot$ Emerson Medeiros Del Ponte $^{3}$
}

Received: 6 November 2020 / Accepted: 13 November 2020 / Published online: 17 November 2020

(C) Sociedade Brasileira de Fitopatologia 2020

We are delighted to deliver the second part of our Special Issue focused on bacterial citrus diseases, which completes the series. This part II is almost entirely dedicated to HLB11 out of 15 articles. We start with the three remaining, all addressing HLB, among which two of them focus on Diaphorina citri (Asian citrus psyllid), the vector of Candidatus Liberibacter asiaticus (CLas), and its relationship with the HLB bacterium. Galdeano and co-workers addressed the impact of CLas on the vector including fitness and behavior, morphology, immune system, metabolism, and microbiome. One of the key findings was that CLas enhances the reproduction and dissemination of psyllids, thus favoring bacteria spread and accumulation on Wolbachia, an endosymbiont that helps to increase the frequency of oviposition by the psyllid female. Transmission mechanism of CLas by the psyllid, as well as the effect of environment on biology, ecology, and behavior of the vector, was discussed by Carmo-Sousa. Factors such as the short acquisition and inoculation time (15 to $30 \mathrm{~min}$ ), the long retention time ( 8 weeks or entire life), the short latency period and long incubation time, and the efficient mobility of winged vector make vector/disease control more difficult but extremely important. However, understanding of transmission mechanisms and insect behavior in the environment is necessary to avoid or stop disease spread.

Another important component, not often considered in disease management programs, is the nutritional status of the host. The effect of nutrient application to maintain or enhance

Alessandra Alves de Souza

desouza@ccsm.br

1 Centro de Citricultura Sylvio Moreira, Instituto Agronômico de Campinas, Cordeirópolis, SP 13490-970, Brazil

2 Citrus Research and Education Center, Department of Microbiology and Cell Science, University of Florida/Institute of Food and Agricultural Sciences, Lake Alfred, FL 33850, USA

3 Departamento de Fitopatologia, Universidade Federal de Viçosa, Viçosa, MG 36570-900, Brazil yield in HLB-affected plants is a controversial topic and is reviewed by Mattos Jr. and collaborators. A critical assessment of literature data on nutrient management practices aimed at maintaining fruit production, and quality is made under HLB-free as well as under conditions of low or high HLB incidence. The impact of the disease and the potential beneficial effects of nutritional programs on plant physiology, cell anatomy, and nutrient imbalance are reviewed and discussed.

The original research articles on HLB cover several aspects. The work by da Silva and collaborators investigated the effect of supplementation with micronutrients $(\mathrm{Zn}, \mathrm{Mn}$, and $\mathrm{Cu}$ ) on HLB-affected plants as well on the CLas acquisition by the vector. Although not reducing CLas titers in plants, micronutrients mitigated the deleterious effects of HLB on starch metabolism. The combination of $\mathrm{Zn}, \mathrm{Mn}$, and $\mathrm{Cu}$, which reduced the acquisition of CLas by adults and especially nymphs of $D$. citri, opens possibilities for reducing disease spread in citrus groves. Another way of reducing HLB incidence is to use rootstock with dwarfing characteristics and that emit few and homogenous flush shoots, thus facilitating psyllid control. Despite the susceptibility of dwarfing "Flying Dragon" rootstock to CLas, Rodrigues and co-workers report reduced levels of HLB incidence and disease progress rate in "Valencia" sweet orange during 8 years of experimentation, encouraging studies using less CLas-susceptible and dwarfing rootstocks. CLas infects all commercial citrus varieties and is present in most of the plant organs, including flower and seed. Even though the vertical transmission of CLas by seeds was not demonstrated so far, Morelli and co-authors showed that seed physiology, germination, and seedlings size for two rootstocks (Rangpur Lime and Swingle citrumelo) were negatively affected by CLas.

Although all commercial citrus varieties are susceptible to CLas, Soratto and collaborators report differential responses to HLB in a progeny of hybrids from the cross of Poncirus trifoliata and Sunki mandarin. The authors found that QTL and eQTL were associated with starch accumulation caused by CLas infection. Molecular mechanisms involved in CLas 
pathogenicity are poorly understood. However, recent in silico analysis of pathogen genomes has shown a repertoire of effector candidates that might play a role in CLas infection and colonization. Using in silico analysis, Granato and collaborators uncovered four potential effector candidates that were highly expressed in HLB symptomatic leaves. The transient expression of effectors in Nicotiana benthamiana enables the researchers to overcome uncultivable characteristic of CLas to investigate the roles of putative effectors in HLB disease.

Epidemiological aspects of HLB were investigated in two articles. In the province of Manipur (Northeast India), Devi and collaborators report that HLB was widely distributed, but with generally low incidence, in regions at 1200 to $1500 \mathrm{~m}$ above the sea level, corroborating reports of low temperatures as more limiting to CLas spread. Alves and co-workers provide novel information on the spatiotemporal spread of HLB at the large, regional, and scale (Minas Gerais state, Brazil). The disease incidence and the rate of spatial spread were higher in South Minas (SM) and Central regions, two major mandarin-producing regions, than at the major orangeproduction region of Triângulo Mineiro (TM). In TM, the orange groves are large, and the vector is controlled intensively; in contrast, production of mandarins is usually in small groves. The study provides important epidemiological parameters to understand and predict epidemics at the large scale. Finally, Lopes-da-Silva and collaborators studied the association of 16S group IX phytoplasma bacteria with HLB symptomatic citrus plants and its occurrence in spontaneous weeds as well as in phloem feeder leafhoppers. The authors found that 16SrIX phytoplasma, but not CLas, is associated with HLB symptomatic plants in the Distrito Federal and Bahia state.

Two research articles on the citrus canker addressed disease control during both pre- and post-harvest periods. Two to four drench applications of plant resistance-inducing compounds, such as acibenzolar-s-methyl (ASM) and thiamethoxam (THIA), resulted in more effective citrus canker control compared with regular foliar application of copper, as reported by
Melo and collaborators. Using a similar approach, Darolt and collaborators showed that exogenous application of acibenzolar-S-methyl (ASM) upregulates the expression of genes related to the biosynthetic pathways of salicylic and jasmonic acids, as well as increases physiological parameters of young sweet orange plants. Zamuner and collaborators showed that a post-harvest decontamination of Xanthomonas citri on citrus fruits surface was successfully achieved using cinnamaldehydebased formulation that was as effective as the traditionally used $\mathrm{NaOCl}$ for $X$. citri decontamination in packinghouse.

The use of tobacco (Nicotiana tabacum) as model plant for Xylella fastidiosa subsp. pauca studies is a good strategy to overcome the long incubation time of this pathogen in citrus plant in addition to high efficiency of artificial inoculation. Using tobacco as a model plant, Pereira and collaborators designed and evaluated a standard area diagram set (SADs) to aid in the visual estimation of severity (percent leaf area affected). The SADs, evaluated at four different laboratories, totaling 50 raters, allowed to generally increase the overall accuracy of the visual estimates of severity of symptoms induced by Xylella fastidiosa subsp. pauca in tobacco, which are not readily discernible and prone to errors of the estimates.

The successful call to an active and relative large community of citrus pathologists/bacteriologists can be confirmed based on our record of 31 articles including 14 literature reviews. Among the diseases, Huanglongbing (HLB) was the predominant disease covered in 19 articles, followed by citrus canker in six articles and CVC with three articles. These numbers highlight the prominence of the bacterial diseases affecting citrus production worldwide. We are confident that the contributions in this two-part Special Issue will provide useful information on different aspects of citrus diseases, further increasing our understanding of the bacterial diseases and improving their management.

Publisher's note Springer Nature remains neutral with regard to jurisdictional claims in published maps and institutional affiliations. 